\title{
Setting the conditions for efficient, robust and reproducible generation of functionally active neurons from adult subventricular zone-derived neural stem cells
}

\author{
D Goffredo ${ }^{1,5}$, L Conti $^{1,5}$, F Di Febo ${ }^{2}$, G Biella ${ }^{2}$, A Tosoni ${ }^{3}$, G Vago ${ }^{3}$, I Biunno ${ }^{4}$, A Moiana ${ }^{1}$, D Bolognini ${ }^{1}$, M Toselli ${ }^{2}$ and E Cattaneo ${ }^{\star, 1}$
}

Although new culture conditions enable homogeneous and long-term propagation of radial glia-like neural stem (NS) cells in monolayer and serum-free conditions, the efficiency of the conversion of NS cells into terminally differentiated, functionally mature neurons is relatively limited and poorly characterized. We demonstrate that NS cells derived from adult mouse subventricular zone robustly develop properties of mature neurons when exposed to an optimized neuronal differentiation protocol. A high degree of cell viability was preserved. At 22 days in vitro, most cells (65\%) were microtubule-associated protein $2^{+}$and coexpressed $\gamma$-aminobutyric acid (GABA), GAD67, calbindin and parvalbumin. Nearly all neurons exhibited sodium, potassium and calcium currents, and $70 \%$ of them fired action potentials. These neurons expressed functional $\mathrm{GABA}_{A}$ receptors, whereas activable kainate, $\alpha$-amino-3-hydroxy-5-methyl-4-isoxazolepropionic acid and $N$-methyl-D-aspartic acid receptors were present in approximately 80,30 and $2 \%$ of cells, respectively. Antigenic and functional properties were efficiently and reliably reproduced across experiments and cell passages (up to 68). This is the first report showing a consistent and reproducible generation of large amounts of neurons from long-term passaged adult neural stem cells. Remarkably, the neuronal progeny carries a defined set of antigenic, biochemical and functional characteristics that make this system suitable for studies of NS cell biology as well as for genetic and chemical screenings.

Cell Death and Differentiation (2008) 15, 1847-1856; doi:10.1038/cdd.2008.118; published online 12 September 2008

The definition of protocols enabling consistent and reproducible isolation, expansion and conversion of neural stem cells (NSCs) into desired mature neuronal phenotypes is a major goal in the field of stem cell technology. NSCs that can be homogeneously propagated in vitro while maintaining high neurogenic potential would represent a valuable tool for studying the mechanisms of neurogenesis and might help to revolutionize regenerative medicine and drug discovery. ${ }^{1}$

Many studies have investigated the properties of NSCs derived from neural tissues and propagated in vitro by means of the neurosphere system. ${ }^{2-5}$ However, this latter system has been reported to have considerable limitations ${ }^{6,7}$ related to the fact that it represents a mixed culture, poorly enriched in NSCs, relative to progenies or other cell types present. ${ }^{8-10}$ Furthermore, different groups have shown that at later passages the neurospheres appear to become more gliogenic, ${ }^{11,12}$ thus indicating that in vitro expansion of
NSCs can alter their biological properties. ${ }^{13,14}$ The mixed character of the neurospheres has also made expression profiling studies difficult to interpret. ${ }^{10,15,16}$

One of the best-characterized, nonimmortalized NSC propagation system is represented by an adherent fibroblast growth factor 2 (FGF-2)-responsive line established from adult rat hippocampus. ${ }^{17}$ Although these cultures are not homogeneous, they can generate neurons and glia with $5-10 \%$ of cells expressing microtubule-associated protein 2 (MAP2)ab or other neuronal markers. ${ }^{17}$ It was also reported that exposure of these cells to retinoic acid followed by the addition of neurotrophins leads to the appearance of different neuronal subtypes. ${ }^{18}$ The treatment of these cells with valproic acid, a histone deacetylase inhibitor, has been shown to increase the fraction of MAP2 ${ }^{+}$cells (up to $60 \%$ ), although the functionality of these new cells has not been fully assessed. ${ }^{19}$ On the other hand, in vitro differentiated adult

\footnotetext{
${ }^{1}$ Department of Pharmacological Sciences and Center for Stem Cell Research, University of Milan, Milan, Italy; ${ }^{2}$ Department of Cellular and Molecular Physiological and Pharmacological Sciences, University of Pavia, Pavia, Italy; ${ }^{3}$ Department of Clinical Sciences 'L Sacco' University of Milan and Pathology Unit, 'L Sacco' Hospital, Milan, Italy and ${ }^{4}$ Department of Medicine, Institute for Biomedical Technologies, National Research Council, Segrate, Milan, Italy

${ }^{*}$ Corresponding author: E Cattaneo, Centre for Stem Cell Research and Department of Pharmacological Sciences, University of Milan, Via Balzaretti 9, 20133 Milano, Italy. Tel: + 390250318333 ; Fax + 39025031 8284; E-mail: elena.cattaneo@unimi.it

${ }^{5}$ These authors contributed equally to this work and should be considered co-first authors.

Keywords: neural differentiation; neural stem cell; adult stem cells; in vitro differentiation; neurones

Abbreviations: AMPA, $\alpha$-amino-3-hydroxy-5-methyl-4-isoxazolepropionic acid; aNS-1 cell, adult SVZ-derived NS cell; AP, action potential; BDNF, brain-derived neurotrophic factor; $\mathrm{Ca}^{2+}$, calcium; CNS, central nervous system; EGF, epidermal growth factor; FGF-2, fibroblast growth factor 2; GABA, $\gamma$-aminobutyric acid; GAD67, glutamate decarboxylase 1; GFAP, glial fibrillary acid protein; MAG, myelin-associated glycoprotein; MAP2, microtubule-associated protein 2; $\mathrm{Na}^{+}$, sodium; $\mathrm{Na}_{\mathrm{v}} 1.2$, type 2 voltage-gated sodium channel; NMDA, N-methyl-D-aspartic acid; NS cells, radial glia-like neural stem cells ; NSC, neural stem cell; OLIG2, oligodendrocyte transcription factor 2; Pax6, paired box gene 6; RC2, intermediate filament-associated protein RC2; SNAP-25, synaptosomal-associated protein 25; SOX2, SRY (sex determining region Y)-box 2; SVZ, subventricular zone; TTX, tetrodotoxin
}

Received 14.11.07; revised 09.6.08; accepted 07.7.08; Edited by G Melino; published online 12.9.08 
mouse SVZ neurosphere-based cultures at passages 5-10 and 20-70 have been shown to contain only a limited number (approximately $11-14 \%$ ) of $\beta 3$-tubulin ${ }^{+}$neurons. ${ }^{20}$

Recently, we reported that adult SVZ cells, isolated and expanded by means of epidermal growth factor (EGF) and FGF-2, can be maintained as adherent stem cell lines in vitro ${ }^{21}$ and express markers of NS cells, a homogeneous population of NSCs with neurogenic radial glia-like properties. ${ }^{22,23}$ We named these cells 'aNS-1 cells'. In this study, we show that approximately $65 \%$ of aNS-1 cells, exposed to an optimized differentiation protocol, progressively develop properties of mature $\gamma$-aminobutyric acid (GABA)ergic neurons over time in vitro. The great majority of these neurons can fire action potentials (APs) and express functionally active receptors of neurotransmitters. On the whole, our data show that aNS-1 cells can efficiently and consistently develop a physiologically mature and functionally active neuronal phenotype in vitro (also after extensive in vitro propagation). Therefore, aNS-1 cells and their mature derivatives might offer a means to examine the role of intrinsic and extrinsic factors in NSC biology and to perform genetic and chemical screenings.

\section{Results}

Long-term-expanded aNS-1 cells exposed to an optimized differentiation procedure generate neurons and astrocytes in vitro. aNS-1 cells derived from adult mice SVZ ${ }^{21}$ have been propagated for up to 68 passages without loss of self-renewal capacity. More than $97 \%$ of long-term expanded aNS-1 cells express markers of radial glia/NS cells (Figure 1a), whereas the fraction of differentiated cells expressing $\beta 3$-tubulin (Figure 1a) or glial fibrillary
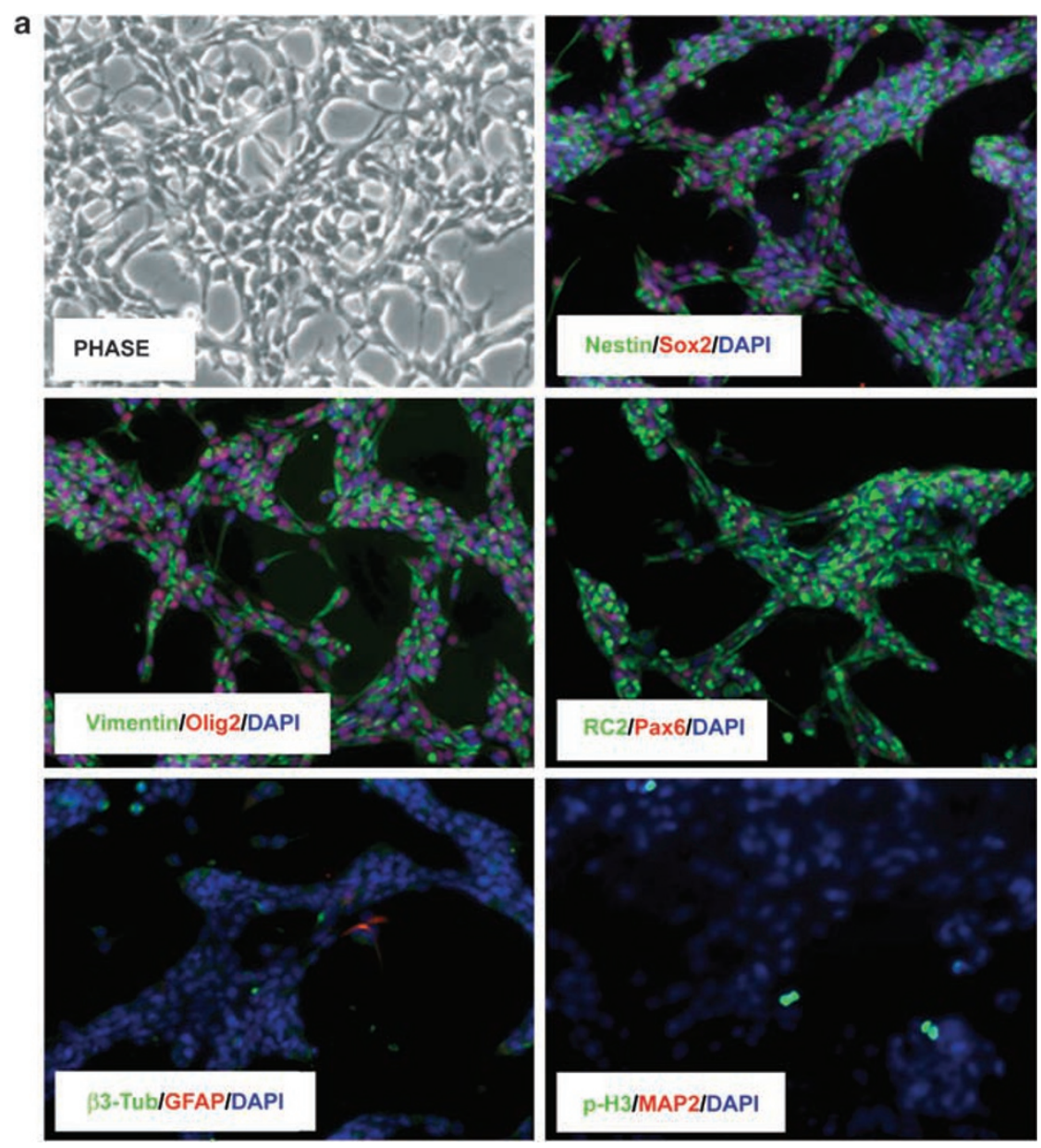

Figure 1 Morphological and antigenic properties of long-term-passaged aNS-1 cells under self-renewing conditions and after exposure to differentiating conditions. (a) In self-renewing conditions, long-term-passaged (up to passage 68) adult SVZ-derived aNS-1 cells maintained their typical morphological and antigenic features. All cells uniformly expressed markers of radial glia and NS cells/neural progenitors such as nestin, Sox2, vimentin, Olig2, Pax6 and RC2. Moreover, aNS-1 cells were mitotically active, as shown by phosphohistone 3 reactivity. Very few $(<2 \%) \beta 3$ tubulin ( $\beta 3$-Tub)-positive, MAP2-positive and GFAP-positive cells were found under proliferating conditions. Nuclei were stained with DAPI (blue). (b) Schematic representation of the four-step neuronal differentiation procedure (see 'Materials and Methods' for details). When induced to differentiate, aNS-1 cells developed complex morphology and cell-cell contacts, the number increasing over time, from (c) 7 DIV to (d) 14 DIV and (e) 22 DIV. (f) After 22 DIV, approximately $80 \%$ of initially plated aNS-1 cells were still alive. RT-PCR analysis revealed, along with aNS-1 cells in vitro maturation (from 0 to 14 DIV), a loss of nestin mRNA levels and an increased expression of neuronal markers such as neurofilaments (NF-M), $\beta 3$-tubulin, synapsin, synaptophysin (synapt), MAP2 and glial markers such as GFAP. (g) There was no detectable expression of the oligodendrocyte marker myelin-associated glycoprotein (MAG) and a faint expression of the neuroblast marker neural cell adhesion molecule (N-CAM), at this stage in vitro. (h) The neuronal maturation is further confirmed by the expression of SNAP-25 mRNA at 14 and 22 DIV 
b

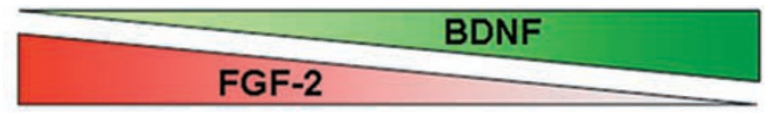

FGF-2
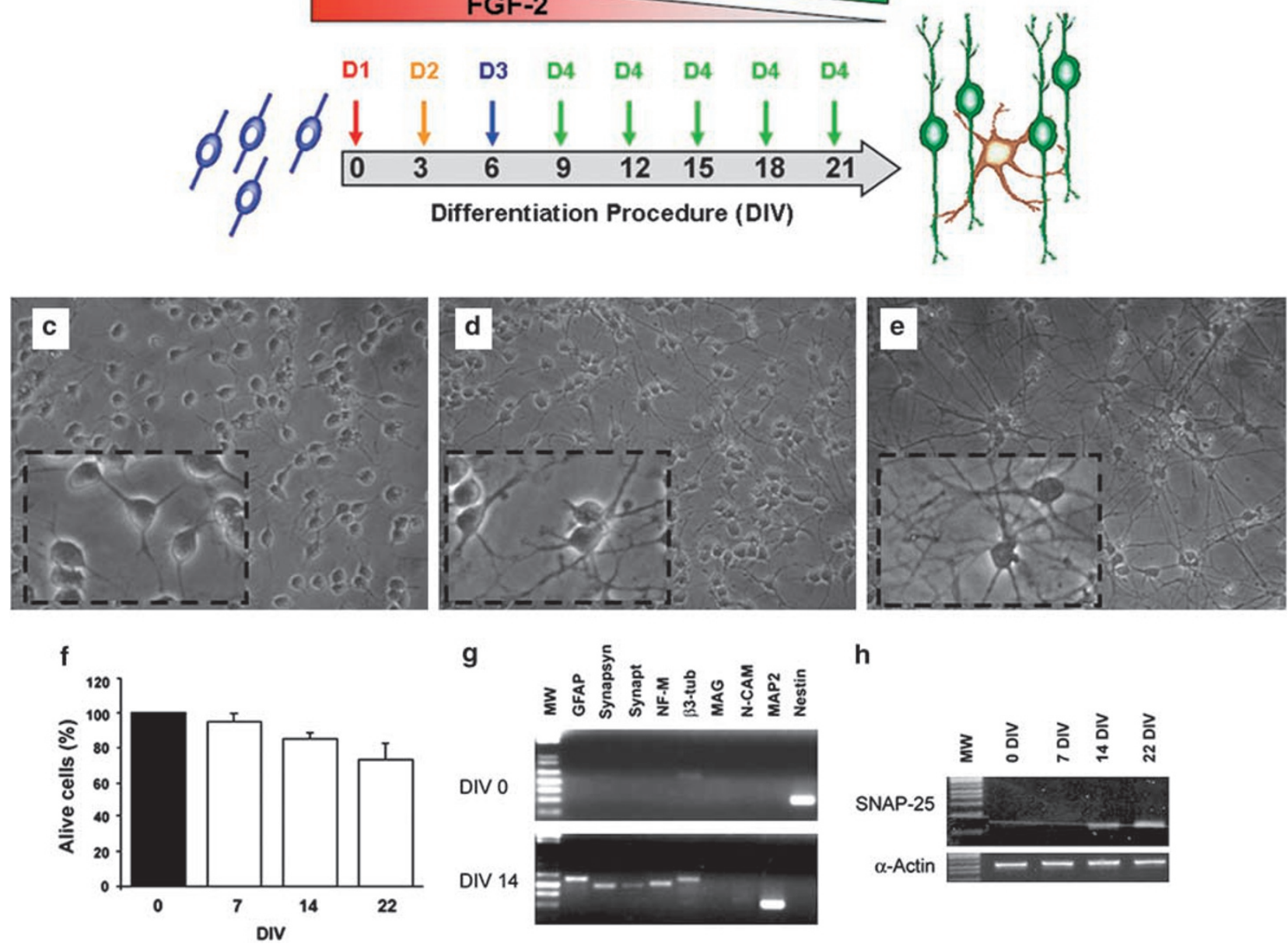

h

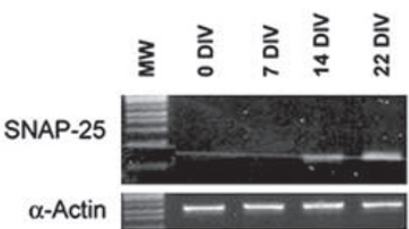

Figure 1 (Continued)

acid protein (GFAP) (not shown) is extremely low (less than $2 \%$ in culture. On the whole, these results indicate that aNS-1 cultures retain their original composition and antigenic properties after extensive expansion in vitro.

To exploit the neurogenic potential of aNS-1 cells, we developed an optimized neuronal differentiation protocol efficiently and reproducibly enabling complete functional and neurochemical maturation of these cells. aNS-1 cells were plated on laminin in FGF-2- and brain-derived neurotrophic factor (BDNF)-supplemented D1 medium and subsequently cultured as described (see 'Materials and Methods' for details; Figure 1b). Under these conditions, aNS-1 cells gradually stopped dividing and progressively developed a neuronal morphology (Figure 1c-e). Importantly, cell viability was well preserved (percentage of live cells among initially plated cells: $96.2 \pm 3.4,87.4 \pm 2.3$ and $73.3 \pm 7.8 \%$ after 7,14 and 22 days in vitro (DIV), respectively; $P<0.001$ by $t$-test; Figure 1f).

The evidence for progressive maturation of aNS-1 cells was confirmed by RT-PCR analysis of the cultures after 14 DIV. This analysis revealed the expression of neuronal and astrocytic markers (Figure 1g). Quantitative real-time
RT-PCR analysis confirmed strong induction of mature neuronal markers after 17 DIV such as TAU (12.57 \pm 3.36 fold induction; data not shown). The low levels of myelinassociated glycoprotein (MAG) mRNA indicate that our differentiation protocol does not favor the generation of oligodendrocyte lineage (Figure 1g), although occasional oligodendrocytes can appear in differentiated culture (data not shown). Also, the expression of synaptic genes such as synaptosomal-associated protein 25 (SNAP-25) gradually increased with in vitro maturation (Figure 1h).

aNS-1 cells efficiently and reproducibly generate neurochemically mature GABAergic neurons. Seeking to characterize the quality and efficiency of differentiation in aNS-1 cultures, we analyzed them by immunocytochemistry at different maturational stages (7, 14 and 22 DIV) and quantitatively evaluated their antigenic properties. Analysis of nestin expression demonstrated a gradual decrease in the number of immunoreactive cells (Figure $2 a$ and $b$ ), with a significant reduction within 7 DIV (self-renewing conditions: 97.2 $\pm 2.7 \%$; 7 DIV: $30.4 \pm 3.8 \% ; P<0.001$ by $t$-test) and $a$ further reduction at 14 DIV $(5.3 \pm 2.2 \% ; P<0.001$ by $t$-test $)$ 
a
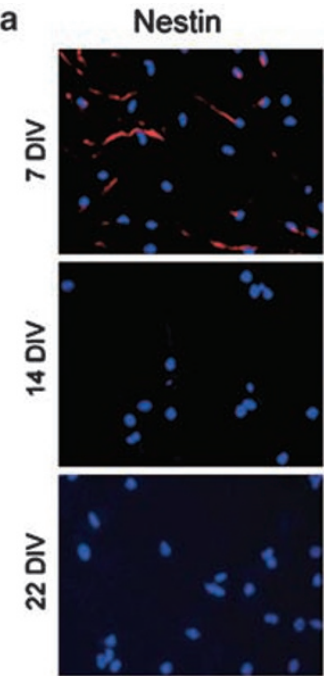

b

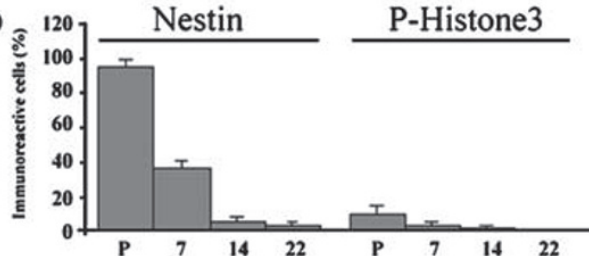

B3-Tubulin
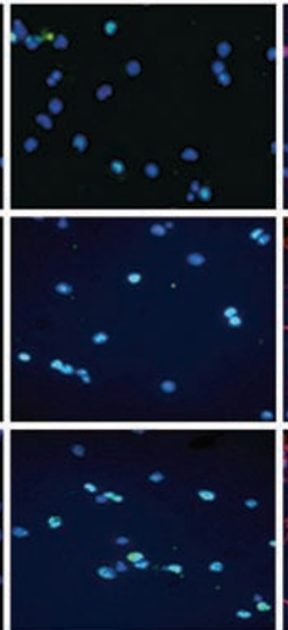

$\therefore$
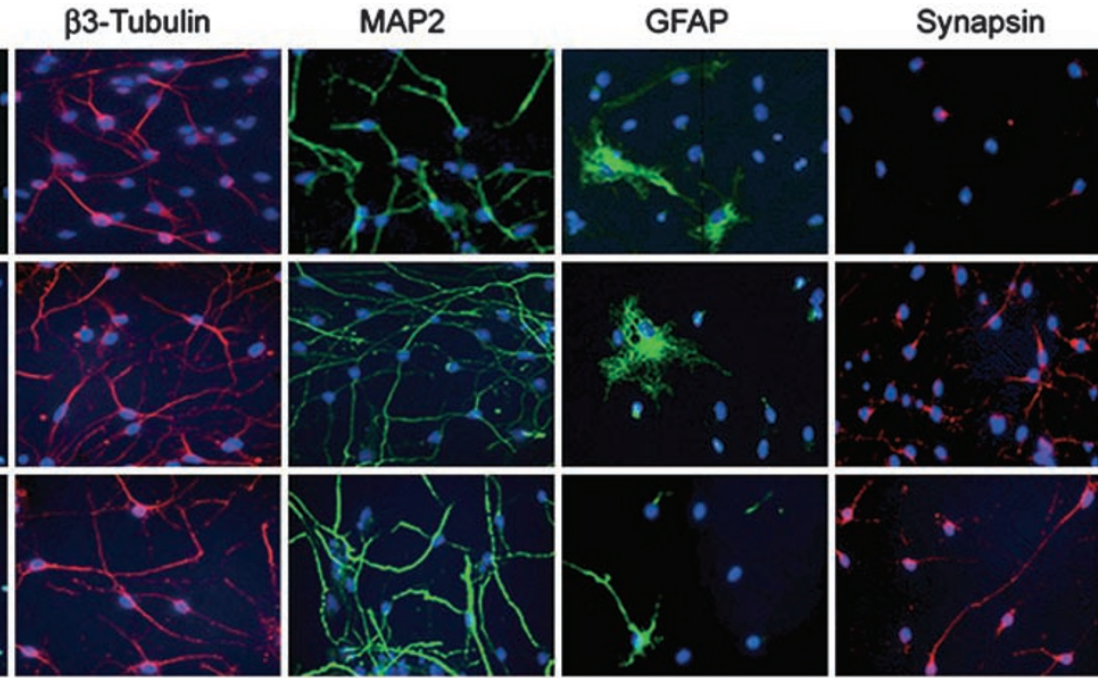

$\beta 3$-tubulin
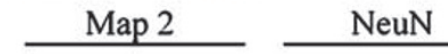

NeuN

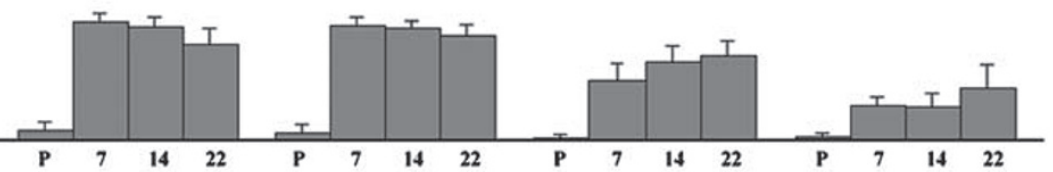

DIV

c

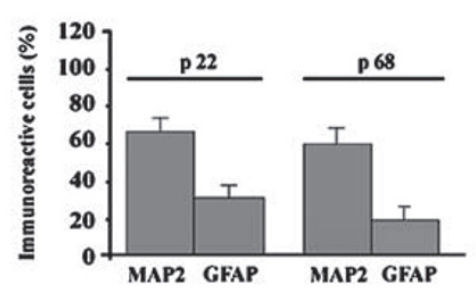

d

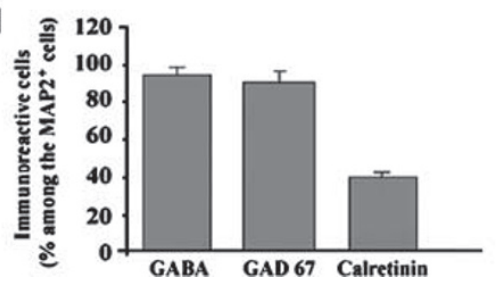

e
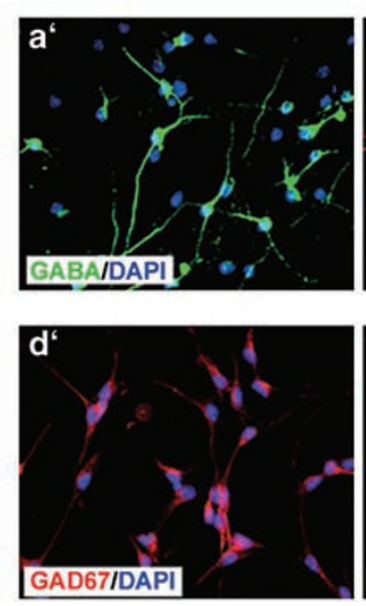
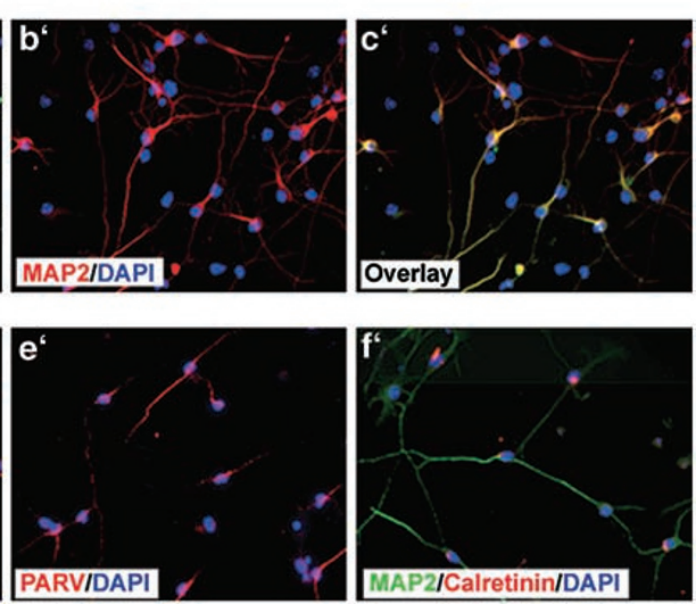

Figure 2 Differentiating aNS-1 cells progressively acquired precise neuronal antigenic properties. (a and b) Downregulation of NS cell markers such as nestin and upregulation of neuronal and glial markers were apparent in aNS-1 cells between 7 and 22 DIV. Under the differentiative conditions, aNS-1 cells did not proliferate, as shown by (b) the reduction in phosphohistone 3 reactivity. (c) aNS-1 cells maintained stable neurogenic potential after long-term in vitro expansion. aNS-1 cells at different passages ( $\mathrm{p} 22$ and p 68) generated comparable percentages of MAP2- and GFAP-positive cells. Moreover, most MAP2-positive (red, eb') differentiating aNS-1 cells expressed antigens typical of GABAergic lineage, such as GABA ( 90\%; green, d, ea'and ec'), the 67-kDa isoform of glutamic acid decarboxylase (GAD67, 90\%; red, d and ed'), parvalbumin ( $\sim 65 \%$; red, ee') and calretinin ( $\sim 40 \%$; red, $\mathbf{d}$ and ef'; MAP2, green, ef'). Nuclei were stained with DAPI (blue; all panels). Syn, synapsin; P-histone3, phosphohistone 3

and 22 DIV $(2.9 \pm 1.8 \% ; P<0.001$ by $t$-test). Similar trends have been observed for other immature markers of aNS-1 cells (Supplementary Figure S1). Consistently, we found that most cells were postmitotic after $7 \mathrm{DIV}$, as indicated by the small fraction of cells with positive staining for pHistone-3 (\% immunoreactive cells: $1.8 \pm 0.6 \%$ ) and with no cells labeled at 14 and 22 DIV (Figure 2b).
We next examined the phenotypic composition of the cultures at the same time points. Most cells had acquired immunoreactive signals for neuronal markers after 7 DIV (Figure 2a and b and Supplementary Figure S1e,h; \% immunoreactive cells: $\beta 3$-tubulin, $67.9 \pm 5.9 \%$; MAP2, $55.4 \pm 5.8 \%$; neuronal nuclei, $40.3 \pm 7.98 \%$ ). Noteworthy, at later time points, the number of cells immunoreactive for these 
neuronal markers did not change significantly (Figure 2a and b and Supplementary Figure S1f-jl), although the immunoreactive signal became more intense (Figure $2 \mathrm{a}$ and Supplementary Figure S1f-j). Cells with a flat astrocyte-like morphology and immunoreactive for GFAP were also present in the cultures after 7 DIV $(21.9 \pm 5.6 \%$ Figure $2 a$ and Supplementary Figure S1e); this was then mostly unchanged throughout the observation period (Figure $2 a$ and $b$ and Supplementary Figure S1i,j).

The concept of in vitro self-maintenance implies that stem cells should retain their original functional properties when grown for extended periods of time. To test whether our stem cell cultures could meet this criterion, we performed eight differentiation experiments in a 12-month period on cells at different passages. We found that the percentage of neurons was maintained with no significant variation across passages (Figure 2c, Table 1). The fraction of GFAP $^{+}$cells was roughly maintained although a reduction was observed in the experiments performed with passage 68 cultures (Table 1). The maturing cultures also showed growth cone-associated protein 43 expression and ultrastructural signs of neurite elongation and neuronal maturation (Supplementary Information; Figures S2 and S3).

These results, together with the analysis of more mature neuronal markers (Figure $2 \mathrm{~d}$ and e), at 14 and 22 DIV confirmed the acquisition of a precise neuronal phenotype over time in vitro. Indeed, almost all of the MAP2 ${ }^{+}$neuronal cells in culture at these stages exhibited GABAergic antigenic properties, as indicated by immunopositivity for GABA (Figure 2e, a'-c') and glutamate decarboxylase 1 (GAD67) (Figure 2e, e'-d'). Real-time RT-PCR analysis further confirmed strong induction of GAD67 (8.43 \pm 1.30 -fold induction; not shown). Interestingly, up to $60 \%$ of neurons at 22 DIV expressed parvalbumin (Figure 2e,e') and up to $40 \%$ expressed calretinin (Figure 2d-f); these antigens were not expressed at earlier time points (not shown). As parvalbumin and calretinin antigens are expressed in the brain in vivo in defined GABAergic sub-populations, it appears that a large proportion of neurons from aNS-1 cells are GABAergic. At 22 DIV, only a fraction $(8.6 \pm 3.1 \%)$ of MAP2 ${ }^{+}$cells coexpressed both these antigens.

Neuronal progeny from the aNS-1 cell line develops sodium and potassium currents and produces action potential. As the expression of voltage-gated sodium $\left(\mathrm{Na}^{+}\right)$channels producing tetrodotoxin (TTX)-sensitive $\mathrm{Na}^{+}$currents should correlate with the capacity to produce APs, we tested the number and potential of the differentiated aNS-1 cells to generate qualitatively identifiable APs.

We first examined the time-dependent appearance of TTXsensitive $\mathrm{Na}^{+}$channels using real-time RT-PCR. After 17 DIV, there was a $4.7 \pm 2.2$-fold induction of the type 2 voltagegated sodium ( $\left.\mathrm{Na}_{\vee} 1.2\right)$ channel relative to the expansion conditions (not shown). The presence of $\mathrm{Na}_{v} 1.2$ protein was confirmed by immunofluorescence staining at different stages of maturation (7, 14 and 22 DIV). Under self-renewing conditions, the cultures did not express $\mathrm{Na}_{\mathrm{v}} 1.2$ channel (not shown), whereas a low level of immunopositivity was exclusively observed in a small number of MAP2 ${ }^{+}$cells after 7 DIV (Figure 3a and Supplementary Figure S5a). At 14 DIV,

Table 1 Quantitative analysis of neuronal and glial marker expression during differentiation of aNS-1 cells at different in vitro passages

\begin{tabular}{|c|c|c|c|c|c|c|c|c|}
\hline DIV & Passage & Nestin & $\beta 3-T u b$ & MAP2 & Synapsin & GABA & GAD67 & GFAP \\
\hline & 19 & $97.18 \pm 1.43$ & $4.03 \pm 1.8$ & $2.98 \pm 3.42$ & ND & ND & ND & $5.49 \pm 1.71$ \\
\hline \multirow[t]{5}{*}{0} & 22 & $96.17 \pm 2.16$ & $3.47 \pm 2.1$ & $2.05 \pm 2.04$ & ND & ND & ND & $1.27 \pm 0.38$ \\
\hline & 35 & $94.05 \pm 2.97$ & $3.86 \pm 2.9$ & $4.18 \pm 3.75$ & ND & ND & ND & $1.18 \pm 1.15$ \\
\hline & 68 & $97.47 \pm 3.03$ & $2.74 \pm 1.3$ & $2.87 \pm 2.62$ & ND & ND & ND & $1.39 \pm 1.42$ \\
\hline & 19 & $36.28 \pm 2.72$ & NA & NA & NA & NA & NA & $\mathrm{NA}$ \\
\hline & 22 & NA & $69.57 \pm 9.24$ & $54.88 \pm 3.07$ & ND & ND & ND & $22.33 \pm 5.47$ \\
\hline \multirow[t]{8}{*}{7} & 33 & $37.34 \pm 5.32$ & NA & NA & ND & ND & ND & NA \\
\hline & 34 & NA & $63.87 \pm 16.03$ & $52.35 \pm 4.4$ & NA & NA & NA & NA \\
\hline & 35 & $39.18 \pm 6.33$ & $60.07 \pm 7.59$ & NA & ND & ND & ND & $22.97 \pm 5.91$ \\
\hline & 40 & NA & NA & $56.41 \pm 6.02$ & NA & NA & NA & $21.67 \pm 4.08$ \\
\hline & 68 & NA & NA & $52.75 \pm 7.65$ & NA & NA & NA & $0.58 \pm 0.57$ \\
\hline & 19 & $6.32 \pm 4.63$ & NA & NA & $51.06 \pm 4.93$ & NA & NA & NA \\
\hline & 22 & NA & $67.27 \pm 5.83$ & $55.43 \pm 4.08$ & NA & NA & NA & $19.05 \pm 4.82$ \\
\hline & 28 & $4.98 \pm 4.36$ & NA & NA & $53.28 \pm 3.62$ & $44.63 \pm 6.23$ & $48.69 \pm 5.47$ & NA \\
\hline \multirow[t]{8}{*}{14} & 33 & $5.33 \pm 4.11$ & NA & NA & $55.66 \pm 1.91$ & $46.45 \pm 9.07$ & $51.01 \pm 8.01$ & $19.68 \pm 6.06$ \\
\hline & 34 & NA & $65.83 \pm 9.05$ & $58.75 \pm 7.03$ & NA & NA & NA & NA \\
\hline & 35 & NA & NA & NA & NA & $48.21 \pm 7.84$ & $53.62 \pm 7.31$ & $22.75 \pm 5.73$ \\
\hline & 40 & NA & $62.66 \pm 12.47$ & $60.14 \pm 8.25$ & NA & $\mathrm{NA}$ & $\mathrm{NA}$ & $\mathrm{NA}$ \\
\hline & 68 & NA & NA & $64.53 \pm 13.38$ & NA & NA & NA & $10.03 \pm 1.05$ \\
\hline & 19 & $1.83 \pm 1.07$ & NA & NA & $59.86 \pm 7.99$ & $45.33 \pm 6.93$ & $54.83 \pm 6.7$ & NA \\
\hline & 22 & NA & $55.93 \pm 5.4$ & $67.03 \pm 0.01$ & NA & NA & NA & $35.21 \pm 5.23$ \\
\hline & 28 & NA & NA & NA & $57.88 \pm 11.4$ & $50.77 \pm 5.94$ & $51.54 \pm 6.56$ & NA \\
\hline \multirow[t]{5}{*}{22} & 33 & $3.19 \pm 6.38$ & NA & NA & $\mathrm{NA}$ & NA & NA & NA \\
\hline & 34 & NA & $56.88 \pm 10.99$ & $62.09 \pm 5.39$ & NA & NA & NA & NA \\
\hline & 35 & $0.72 \pm 4.08$ & NA & NA & $63.15 \pm 4.69$ & $48.62 \pm 5.37$ & $52.38 \pm 4.28$ & $33.39 \pm 1.91$ \\
\hline & 40 & NA & $65.28 \pm 12.26$ & $64.12 \pm 8.56$ & NA & $\mathrm{NA}$ & NA & $34.73 \pm 3.68$ \\
\hline & 68 & NA & $57.90 \pm 4.87$ & $59.07 \pm 8.30$ & NA & NA & NA & $19.18 \pm 1.48$ \\
\hline
\end{tabular}

The number of cells immunoreactive for immature, neuronal and glial antigens was evaluated by immunocytochemistry in cultures in self-renewing conditions or exposed to neuronal differentiation conditions for 7, 14 and 22 DIV. aNS-1 cultures at different passages were considered to test the neurogenic stability of the cell line and the robustness of the neuronal differentiation procedure. Data were confirmed in three independent experiments. Values are given as mean \pm S.D. 

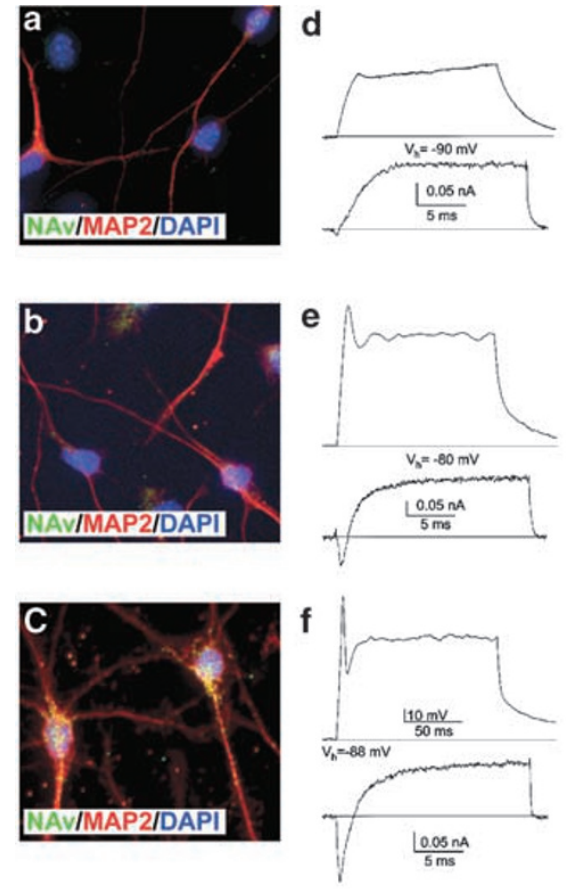

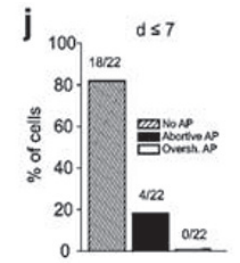

h

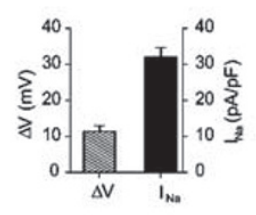

$\mathbf{k}$

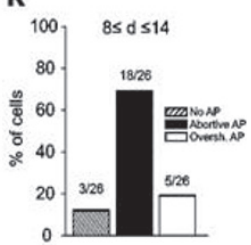

I
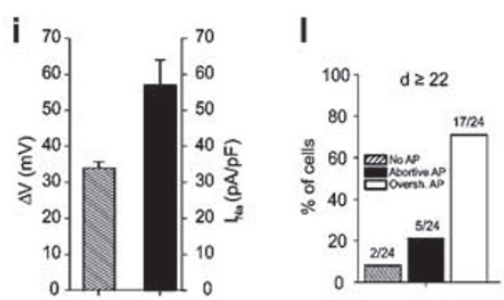

Figure 3 Impulse variability and $\mathrm{Na}^{+}$current amplitude in aNS-1 cells during neuronal differentiation. (a-c) Increased levels of $\mathrm{Na}^{+}$channel protein (green) in aNS-1 cells after (a) 7, (b) 14 and (c) 22 DIV. Neurons were revealed by MAP2 immunoreactivity (red; a-c), and nuclei were labeled with DAPI (blue; a-c). (d-f) Upper traces: voltage responses under current-clamp conditions from three separate aNS-1 cells at different stages of neuronal differentiation (7, 14 and 22 DIV, respectively); the cells were stimulated with rectangular current pulses of $180 \mathrm{pA}$. Lower traces: whole-cell current responses from the same three cells during voltage-clamp recording $\left(V_{\text {test }}=-10 \mathrm{mV}\right)$. $(\mathbf{g}-\mathbf{i})$ Correlation between regenerative voltage response and $\mathrm{Na}^{+}$current density amplitude at (g) less than 7 DIV $(n=13)$, (h) between 8 and 14 DIV ( $\left.n=15\right)$ and (i) at more than 22 DIV $(n=7)$. The magnitude of the regenerative voltage response was quantified by calculating the difference ( $\Delta V$ between the peak of the response and the extrapolated baseline, as shown in the inset to $(\mathbf{g})$. (j-I) Bar graphs showing the percentages of cells producing no APs (no AP), producing abortive APs (abortive AP) and producing overshooting APs (oversh. AP) following application of the differentiating agents for (j) 7 days or fewer, (k) for 8 to 14 days and (I) for 22 or more days

the large majority (up to $85 \%$ ) of $\mathrm{MAP2}^{+}$cells was immunopositive, although with different levels (Figure $3 \mathrm{~b}$ and Supplementary Figure S5a). At 22 DIV, almost all MAP2 ${ }^{+}$ cells displayed a uniform and intense $\mathrm{Na}_{v} 1.2$ immunoreactive signal (Figure 3c and Supplementary Figure S5a).

Whole-cell patch-clamp technique showed a close correlation between the immunocytochemical observations and the appearance of voltage-gated $\mathrm{Na}^{+}$currents recorded under voltage-clamp conditions. Figure $3 \mathrm{~d}-\mathrm{f}$ (lower traces) shows sample records of the total inward and outward currents elicited at a test potential of $-10 \mathrm{mV}$ from a holding voltage of $-90 \mathrm{mV}$ from three aNS-1 cells exposed to differentiating conditions for 7 (Figure 3d), 14 (Figure 3e) and 22 DIV (Figure 3f), respectively. The amplitude of the inward $\mathrm{Na}^{+}$current was negligible after 7 DIV, but subsequently increased dramatically, suggesting that the development of voltage-gated $\mathrm{Na}^{+}$conductance is more gradual than that of delayed rectifier potassium conductances, which were fully expressed in the early DIV (see Supplementary Figure S4). The inward current could be completely blocked by the application of the selective voltage-gated $\mathrm{Na}^{+}$channel blocker TTX (see Supplementary Figure S5). Significantly, upon switching from voltage clamping to current clamping and during stimulation with rectangular current pulses, the same cells generated quite different voltage responses (Figure $3 d-f$, upper tracings). Indeed, although the aNS-1 cells exposed to the differentiating conditions for 7 DIV showed almost no regenerative response, cells treated for 14 DIV exhibited a regenerative but abortive response, and only the cells that had been differentiated for 22 DIV produced an overshooting AP. The histograms in Figure $3 \mathrm{~g}-\mathrm{i}$ show the development of a graded AP and $\mathrm{Na}^{+}$current amplitudes at different stages of aNS-1 cell differentiation. The data were ordered into three samples according to the number of DIV. The $I_{\mathrm{Na}}$ increased with increasing DIV, as expected based on the progressive increase in $\Delta V$ values (Figure 3). Figure 3j-I shows the percentage of neuronal cells producing overshooting and abortive APs or no APs as a function of the number of DIV. The highest percentage of cells $(70.9 \%)$ producing overshooting APs and the lowest percentage of cells not producing APs (8.3\%) were observed at 22 or more DIV (Figure 3I).

The maturing cultures also showed the expression of voltage-activated calcium $\left(\mathrm{Ca}^{2+}\right)$ channels producing $\mathrm{Ca}^{2+}$ currents (Supplementary Information; see Supplementary Figure S6).

Overall, these data indicate that changes in $\mathrm{Na}^{+}$channels closely correlated with the ability of differentiating aNS-1 cells to generate APs. Moreover, our protocol yielded an unprecedented proportion of neurons firing APs from long-termpassaged adult NSCs.

aNS-1-derived neurons express functional receptors for GABA and glutamate. To further explore the potential usefulness of our system for studying the biochemical and 
pharmacological properties of relevant neurotransmitter systems, we assessed whether ligand-gated channels that could be activated by inhibitory or excitatory neurotransmitters were present in these cells. RT-PCR performed at 17 DIV demonstrated the expression of $\mathrm{GABA}_{\mathrm{A}}$ receptors (Figure $4 \mathrm{a}$ ) and the absence of the $\mathrm{GABA}_{\mathrm{B}}$ transcript (not shown). Presence of the $\mathrm{GABA}_{\mathrm{A}}$ receptor at $17 \mathrm{DIV}$ was confirmed by immunocytochemistry (see Supplementary Figure S7a) and real-time RT-PCR, which demonstrated a 22-fold induction of $\mathrm{GABA}_{A}$ receptor transcript relative to cultures in self-renewing conditions (Figure 4a). Appearance of the $\mathrm{GABA}_{\mathrm{A}}$ immunoreactive cells progressively increased with time (see Supplementary Figure S7b).

To verify functional activation of this receptor system, we applied GABA (Figure 4b, upper left trace) or the selective $\mathrm{GABA}_{\mathrm{A}}$ agonist muscimol $(20 \mu \mathrm{M})$ and selective $\mathrm{GABA}_{\mathrm{A}}$ antagonist bicuculline (Figure $4 \mathrm{~b}$, right traces) to cultures at 17 DIV. Figure 4b shows sample traces of ionic currents elicited in neuronal differentiated aNS-1 cells during application of these molecules. As summarized in Figure $4 \mathrm{c}$, the GABAergic response was negligible at less than 11 DIV $(n=8)$, whereas the number of responding cells increased together with the differentiation time, increasing from $13 \%$ between 11 and 14 DIV to $57 \%$ in cells measured after this period, between 15 and 22 DIV. After more than 15 DIV, approximately half of the cells responded to muscimol application, and the average ionic current was $-127 \pm$ $38 \mathrm{pA}$, confirming the presence of functional $\mathrm{GABA}_{\mathrm{A}}$ receptors. In contrast, the selective $\mathrm{GABA}_{\mathrm{B}}$ agonist baclofen (lower left trace) had a negligible effect after 14 DIV, further confirming the absence of $\mathrm{GABA}_{\mathrm{B}}$ receptors.

We next evaluated the presence and the functionality of the main classes of glutamatergic receptors. Real-time RT-PCR showed an increase in the expression of $\alpha$-amino-3-hydroxy5-methyl-4-isoxazolepropionic acid (AMPA) (6.59 \pm 1.62-fold induction), kainate (4.63 \pm 0.56 -fold induction) and $N$-methylD-aspartic acid (NMDA) (14.52 \pm 1.96 -fold induction) receptor transcripts after 17 DIV (Figure 4d). Immunofluorescence analysis at 17 DIV demonstrated the presence of cells immunoreactive for the NMDA R1 receptor (see Supplementary Figure S7c). The fraction of NMDA R1 immunoreactive cells increased with in vitro maturation: immunoreactivity was absent under self-renewing conditions and before 9 DIV, whereas at 10 and 17 DIV, $9.6 \pm 0.5$ and $27.1 \pm 4.3 \%$ of cells, respectively, were immunoreactive for NMDA R1 (see Supplementary Figure S7d). Figure $4 \mathrm{e}$ shows ionic currents elicited during the application of the glutamatergic agonists AMPA (left trace), kainate (middle trace) and NMDA (right trace). In general, sizeable glutamatergic responses could be elicited only after 15 DIV. Remarkably, as shown in Figure 4f, among the cells with a neuronal-like morphology, approximately $30 \%$ generated a sizeable AMPA response, a

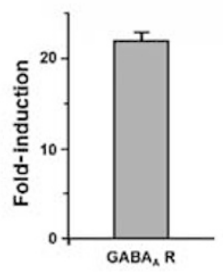

d

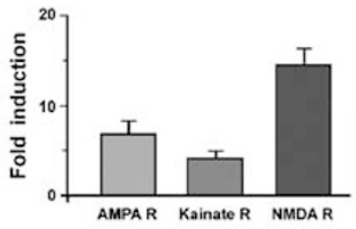

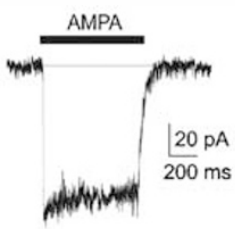
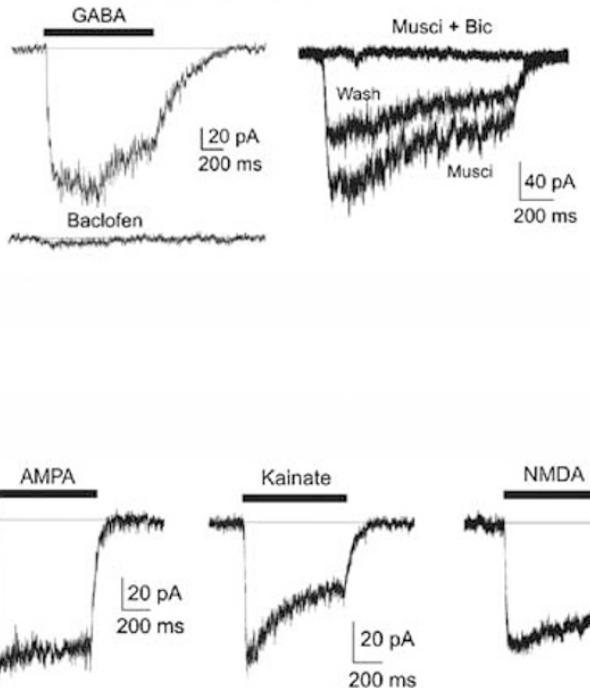

e

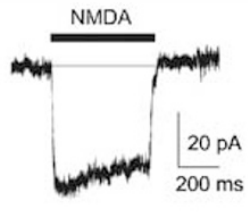

C
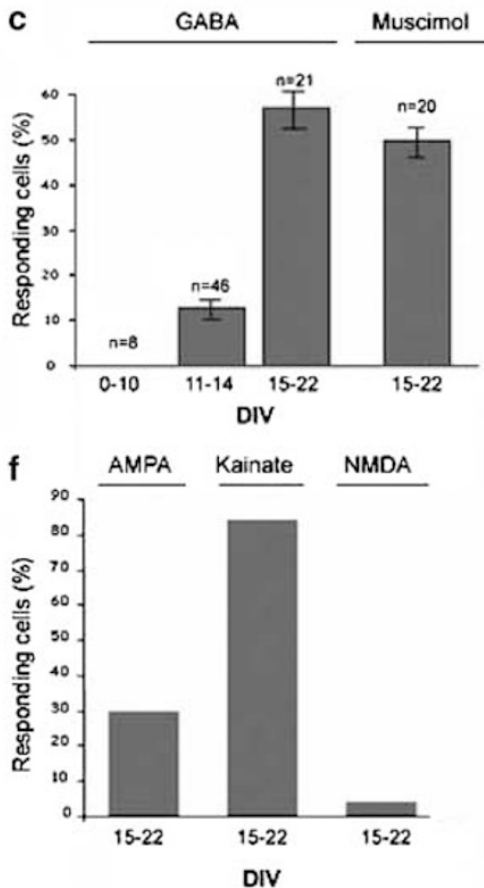

Figure 4 Activation of ligand-gated channel currents in differentiating aNS-1 cells. (a) RT-PCR analysis demonstrated strong induction of $G_{A B A} A_{A}$ receptor $\left(G A B A_{A} R\right.$ ) transcript after 17 DIV relative to self-renewing conditions. (b) Sample traces showing the effects of GABAergic agonists. Left traces: application of $20 \mu \mathrm{M}$ GABA (upper trace) and $20 \mu \mathrm{M}$ baclofen (lower trace) to the same cell at a holding potential of $-80 \mathrm{mV}$. Right traces: application of $20 \mu \mathrm{M}$ muscimol (Musci) and muscimol plus bicuculline (Bic) $(50 \mu \mathrm{M})$ to the same cell at a holding potential of $-80 \mathrm{mV}$. (c) Percentage of cells responding to $\mathrm{GABA}_{\mathrm{A}}$ receptor agonists $(20 \mu \mathrm{M})$ at different stages of cell differentiation. (d) Quantitative real-time PCR showing the induction of main ligand-gated glutamatergic channels in aNS-1-derived neurons after 17 days of differentiation relative to selfrenewing conditions. Transcripts of AMPA receptors (AMPA R; Glu-R2), kainate receptors (kainate R; Glu-R5) and NMDA receptors (NMDA r; NMDA-R1) were markedly induced after 17 DIV (7.71 \pm 1.83 -, $4.60 \pm 0.57$ - and 14.52 \pm 8.27 -fold induction, respectively). (e) Sample traces showing the responses of three aNS-1 cells to application of the selective glutamatergic agonists $(100 \mu \mathrm{M})$ NMDA, AMPA and kainate (holding potential $-80 \mathrm{mV}$ ). Note that the extracellular saline containing NMDA also contained glycine $10 \mu \mathrm{M}$ and was free of $\mathrm{Mg}^{2+}$. (f) Percentage of neuronal cells responding to the glutamate receptor agonists AMPA, kainate and NMDA (100 $\left.\mu \mathrm{M}\right)$ between 15 and $22 \mathrm{DIV}$ 
approximately $84 \%$ generated a kainate response and approximately $2 \%$ generated NMDA response. Collectively, these data indicate that a considerable fraction of differentiated neurons derived from aNS-1 cells carries functionally active glutamate and GABA receptors further indicating the presence of mature neurons in the differentiated cultures. The functional maturation of these neurons was also demonstrated by the capacity of the cultures to incorporate and release GABA in response to specific stimuli (see Supplementary Information; Supplementary Figure S8).

\section{Discussion}

The major finding of this study is that the exposure of aNS-1 cells to in vitro fully defined differentiation conditions efficiently yields functionally mature neurons. In particular, we have shown that even after long-term in vitro expansion, aNS-1 cells retain their capacity to yield a high percentage of MAP2 ${ }^{+}$ neurons with a well-defined GABAergic phenotype able to fire APs and expressing functional neurotransmitter receptors.

\section{Neuronal differentiation of adult SVZ-derived aNS-1} cells. Several recent studies have shown that NSCs that endure in different regions of the adult central nervous system (CNS) can be expanded in vitro. Also, a number of protocols describing their neuronal differentiation potential already exist. These expandable sources of adult NSCs, besides representing a source of donor tissue for the potential achievement of neuroreconstruction after neural transplantation, ${ }^{24}$ can also offer insight into the mechanisms of neuronal maturation and opportunities for screening novel CNS pharmaceuticals. ${ }^{21}$ Presently, however, such applications are limited by the incomplete biological characterization of NSC cultures with regard to cellular composition, long-term stability and the capacity to generate large numbers of well-characterized mature neurons.

Generally, in vitro studies designed to assess the extent of adult neurogenesis focus on SVZ-derived neurospheres at early passages (passages 2-7). However, neurospheres have considerable limitations as a stem cell propagation system as loss of neurogenic potential is observed over multiple passages. ${ }^{11,25}$ Nonetheless, neurospheres have been a precious tool for revealing some properties of NSCs, and their ability to release beneficial factors has generated valuable results in experimental transplantation paradigms. ${ }^{26}$

In contrast, NS cells offer the possibility of expanding a nearly pure adherent neurogenic radial glia-like stem cell population in vitro. ${ }^{22,23}$ In particular, the major advances of the aNS-1 cell system are its reduced complexity and stable longterm growth. ${ }^{21}$ To our knowledge, one of the few monolayer systems not genetically immortalized and derived from the adult rodent brain, is represented by progenitors derived from the adult rat hippocampus. ${ }^{17}$ The neuronal differentiation efficiency in vitro of these cells is limited to $10 \%,{ }^{17,18}$ but increases to $60 \%$ with exposure to valproic acid; ${ }^{19}$ also, this system has been valuable for elucidating the role of astroglial cocultures. $^{27}$

Unlike rat and mouse adult hippocampal progenitor cells, aNS-1 cells are derived from mouse SVZ. This might contribute to important differences in the populations. For example, aNS-1 cells are cultured in monolayer in the presence of FGF-2 and EGF. Our observations show that exposure to fully defined serum-free conditions is sufficient for sustaining efficient neurogenesis and leads to the development of a large number of MAP2 ${ }^{+}$neuronal cells with mature morphological and antigenic properties. It is worth noting that under these defined conditions, the generation of neurons is extremely robust and reproducible across passages in vitro (Table 1), a feature that is crucial for molecular, biological and screening applications.

Even though we never observed modifications in the behavior of aNS-1 cells that might be indicators of cellular transformation, a preliminary karyotypic analysis performed on aNS-1 cells at passage 40 has enlightened the presence of chromosomal abnormalities in more than $50 \%$ of the cells (not shown). In the literature, there are contrasting data on chromosomal stability of nonimmortalized NSC lines and some data indicate that, at least for adult mouse SVZ stem cells in vivo, chromosomal abnormalities might actually be somehow expected. ${ }^{28-33}$ Although preliminary, these results indicate that the chromosomal instability described by a number of authors for both adult mouse SVZ-derived neurosphere ${ }^{8,20}$ and adult rat hippocampal progenitor ${ }^{17}$ cultures is also present in aNS-1 cells. Further analyses are required to better define when these aberrations arise in the aNS cultures and how they evolve with time.

Neurochemical properties of neurons derived from aNS-1 cells. Neurons derived from aNS-1 cells express a set of markers specific to fully competent GABAergic neurons. Previous studies reported the in vitro differentiation of neural precursors into GABAergic phenotypes. $^{34}$ It has been suggested that the GABAergic phenotype is the default differentiation pathway for neuronal precursor cells. ${ }^{35,36}$ However, in nearly all cases, GABAergic lineage was defined by either GAD67 or GABA immunoreactivity, which is not thought to indicate synaptic GABAergic function. In contrast, the aNS-1 cell line can be readily induced to differentiate so as to produce cells with a relatively complete GABAergic phenotype. These cells express GAD67, produce GABA and release GABA in response to an appropriate stimulus. As aNS-1 cells are stable in vitro and can be readily and efficiently induced to undergo GABAergic differentiation in vitro, these cells may provide a useful tool for studies of GABAergic neuronal differentiation and in models of neurodegenerative and neurocognitive disease related to GABAergic function.

\section{Electrophysiological properties of aNS-1-derived} neurons. Our results show that significant electrophysiological changes occur between the early and late stages of neuronal maturation of aNS-1 cells. We found an increase in $\mathrm{Na}^{+}$current density in strict correlation with an increase in the proportion of cells generating overshooting APs.

A substantial increase in the number of cells expressing $\mathrm{Na}^{+}$channel proteins and producing voltage-gated $\mathrm{Na}^{+}$ currents with features similar to those observed in mature neurons was observed only after prolonged exposure to neuronal differentiation conditions. 
Similar behavior was observed for voltage-gated $\mathrm{Ca}^{2+}$ channels and the underlying ionic currents. These findings are consistent with the idea that these neurons have proper neuronal functionality ${ }^{37-40}$ and further support the usefulness of this system for studying physiological events that occur during the maturation of neuronal electrophysiology.

Most importantly, to our knowledge, this is the first description of long-term-expanded NSCs generating such a high percentage (65\% after 15 DIV) of electrophysiologically mature neurons. The neurotransmitter receptors present in differentiated aNS- 1 cells are functional, correctly responding to specific agonists and antagonists, and offer a possible permanent and reliable in vitro source of stem cell-derived neurons for biochemical and pharmacological studies.

\section{Conclusion}

The results presented here provide evidence that aNS-1 cells can generate a population of antigenically mature and electrophysiologically active neurons. The homogeneity and neurogenic stability of this cell system enabled us to systematically and reproducibly demonstrate progressive, efficient, robust and reliable conversion of aNS-1 cell population into neurochemically mature and electrophysiologically active GABAergic neurons of the adult mammalian brain. We believe that aNS-1 cells and their mature derivatives offer an important tool for characterizing the regulatory role of intrinsic and extrinsic factors in NSC biology as well as a system suitable for genetic and chemical screens.

\section{Materials and Methods}

Differentiation of adult SVZ-derived aNS-1 cells. The adult SVZderived NS cell line aNS-1 was derived from the SVZ of a 2-month-old wild-type (CD1 strain) mouse, as described earlier. ${ }^{21}$ In this study, aNS-1 cells between passages 15 and 70 were used. Detailed protocols for routine handling of aNS-1 cells are available elsewhere (see Supplementary Information). ${ }^{21,22}$

The procedure for growing and terminally differentiating aNS-1 cells described here was developed entirely under serum-free conditions. The neuronal differentiation procedure was optimized using four steps specific media that contain DMEM/F12/NeuroBasal in specific ratios (D1-D4 media) and definite supplements (Figure 1b). Briefly, aNS-1 cells were plated (plating density: $5 \times 10^{4}$ $7.5 \times 10^{4} \mathrm{cells} / \mathrm{cm}^{2}$ ) on laminin-coated (laminin from Gibco, Invitrogen) plastic (Corning, Celbio) or glass coverslips in D1 medium consisting of DMEM/F12 medium (Invitrogen) containing 0.5\% N2 (Invitrogen) and 1\% B27 supplements (Invitrogen) and supplemented with FGF-2 (10 ng/ml; PeproTech, Tebu-Bio) and BDNF $\left(20 \mathrm{ng} / \mathrm{ml}\right.$; PeproTech, Tebu-Bio) and cultured for 3 days at $37^{\circ} \mathrm{C}$. The cultures were shifted then to D2 medium composed of a 1:1 mix of DMEM/F12 and neurobasal media (Invitrogen) containing $0.5 \%$ N2 and 1\% B27 supplements and containing FGF-2 $(6.7 \mathrm{ng} / \mathrm{ml})$ and BDNF $(30 \mathrm{ng} / \mathrm{ml})$, for 3 days. Subsequently, the cultures were exposed to D3 medium made of a 1:3 mix of DMEM/F12 and neurobasal media containing $0.5 \% \mathrm{~N} 2$ and $1 \%$ B27 reagents supplemented with FGF-2 $(6.7 \mathrm{ng} / \mathrm{ml})$ and BDNF $(30 \mathrm{ng} / \mathrm{ml})$ for 3 days. For terminal differentiation, the cultures were exposed to D4 medium composed of a 1:3 mix of DMEM/F12 and neurobasal media containing $0.5 \% \mathrm{~N} 2$ and $1 \%$ B27 supplements and containing FGF-2 $(5 \mathrm{ng} / \mathrm{ml})$ and BDNF $(30 \mathrm{ng} / \mathrm{ml})$. The cells were maintained under these conditions for an additional 7-14 days; the medium was partially changed every 3 days.

Total RNA extraction, cDNA production and RT-PCR/quantitative real-time PCR analyses. Total RNA extraction, retrotranscription and PCRs were performed using standard procedures (see 'Supplementary Information' for details). Primer sequences and amplicon sizes for RT-PCR and quantitative realtime PCR are shown in Table S1 and S2, respectively.
Immunocytochemistry and electron microscopy. Immunocytochemistry and electron microscopy analyses were performed using standard protocols (see 'Supplementary Information' for details). Supplementary Table S3 provides a list of the primary antibodies used in this study.

Electrophysiology. Electrophysiology studies were performed as described earlier. $^{22}$ Details of the methodological procedures can be found in the Supplementary Information.

Acknowledgements. This research was supported by funding from EuroStemCell (FP6, EU) and from Cariplo NOBEL to EC and from MIUR (project 2005051740) to LC and MT and partially from NEUROscreen (FP6, EU) to LC and IB. We thank Giulio Simonutti for helpful technical assistance and BioRep for the support on karyotyping experiments.

Competing interests: EC holds equity in Dialectica srl, a spin-off company of the University of Milano that has submitted a patent application entitled 'Neural stem cells and differentiation method'; inventors: E Cattaneo, L Conti, D Goffredo and E Reitano (Universita' degli Studi di Milano, Milan-Italy); EU application no. EP 06025098.8, submitted on 11 December 2006. LC is a consultant to Dialectica srl.

1. Gottlieb DI. Large-scale sources of neural stem cells. Annu Rev Neurosci 2002; 25: 381-407.

2. Reynolds BA, Tetzlaff W, Weiss S. A multipotent EGF-responsive striatal embryonic progenitor cell produces neurons and astrocytes. J Neurosci 1992; 12: 4565-4574.

3. Vescovi AL, Parati EA, Gritti A, Poulin P, Ferrario M, Wanke E et al. Isolation and cloning of multipotential stem cells from the embryonic human CNS and establishment of transplantable human neural stem cell lines by epigenetic stimulation. Exp Neurol 1999; 156: $71-83$

4. Gritti A, Parati EA, Cova L, Frolichsthal P, Galli R, Wanke E et al. Multipotential stem cells from the adult mouse brain proliferate and self-renew in response to basic fibroblast growth factor. J Neurosci 1996; 16: 1091-1100.

5. Reynolds BA, Weiss S. Generation of neurons and astrocytes from isolated cells of the adult mammalian central nervous system. Science 1992; 255: 1707-1710.

6. Jessberger S, Clemenson Jr GD, Gage FH. Spontaneous fusion and nonclonal growth of adult neural stem cells. Stem Cells 2007; 25: 871-874.

7. Singec I, Knoth R, Meyer RP, Maciaczyk J, Volk B, Nikkhah G et al. Defining the actual sensitivity and specificity of the neurosphere assay in stem cell biology. Nat Methods 2006; 3: $801-806$

8. Morshead CM, Benveniste $P$, Iscove NN, van der Kooy D. Hematopoietic competence is a rare property of neural stem cells that may depend on genetic and epigenetic alterations. Nat Med 2002; 8: 268-273.

9. Reynolds BA, Rietze RL. Neural stem cells and neurospheres-re-evaluating the relationship. Nat Methods 2005; 2: 333-336.

10. Suslov ON, Kukekov VG, Ignatova TN, Steindler DA. Neural stem cell heterogeneity demonstrated by molecular phenotyping of clonal neurospheres. Proc Natl Acad Sci USA 2002; 99: 14506-14511.

11. Sun YE, Martinowich $\mathrm{K}$, Ge W. Making and repairing the mammalian brain-signaling toward neurogenesis and gliogenesis. Semin Cell Dev Biol 2003; 14: 161-168.

12. Winkler C, Fricker RA, Gates MA, Olsson M, Hammang JP, Carpenter MK et al. Incorporation and glial differentiation of mouse EGF-responsive neural progenitor cells after transplantation into the embryonic rat brain. Mol Cell Neurosci 1998; 11: 99-116.

13. Kelly CM, Zietlow R, Dunnett SB, Rosser AE. The effects of various concentrations of FGF2 on the proliferation and neuronal yield of murine embryonic neural precursor cells in vitro. Cell Transplant 2003; 12: 215-223.

14. Zhou FC, Kelley MR, Chiang YH, Young P. Three to four-year-old nonpassaged EGFresponsive neural progenitor cells: proliferation, apoptosis, and DNA repair. Exp Neurol 2000; 164: 200-208.

15. Bonnert TP, Bilsland JG, Guest PC, Heavens R, McLaren D, Dale C et al. Molecular characterization of adult mouse subventricular zone progenitor cells during the onset of differentiation. Eur J Neurosci 2006; 24: 661-675.

16. Navarro-Galve B, Martinez-Serrano A. 'Is there any need to argue' about the nature and genetic signature of in vitro neural stem cells? Exp Neurol 2006; 199: 20-25.

17. Palmer TD, Takahashi J, Gage FH. The adult rat hippocampus contains primordial neural stem cells. Mol Cell Neurosci 1997; 8: 389-404.

18. Takahashi J, Palmer TD, Gage FH. Retinoic acid and neurotrophins collaborate to regulate neurogenesis in adult-derived neural stem cell cultures. J Neurobiol 1999; 38: 65-81.

19. Hsieh J, Nakashima K, Kuwabara T, Mejia E, Gage FH. Histone deacetylase inhibitionmediated neuronal differentiation of multipotent adult neural progenitor cells. Proc Natl Acad Sci USA 2004; 101: 16659-16664.

20. Foroni C, Galli R, Cipelletti B, Caumo A, Alberti S, Fiocco R et al. Resilience to transformation and inherent genetic and functional stability of adult neural stem cells ex vivo. Cancer Res 2007; 67: 3725-3733. 
21. Pollard SM, Conti L, Sun Y, Goffredo D, Smith A. Adherent neural stem (NS) cells from fetal and adult forebrain. Cereb Cortex 2006; 16 (Suppl 1): i112-i120.

22. Conti L, Pollard SM, Gorba T, Reitano E, Toselli M, Biella G et al. Niche-independent symmetrical self-renewal of a mammalian tissue stem cell. PLoS Biol 2005; 3: e283.

23. Pollard SM, Conti L. Investigating radial glia in vitro. Prog Neurobiol 2007; 83: 53-67.

24. Conti L, Reitano E, Cattaneo E. Neural stem cell systems: diversities and properties after transplantation in animal models of diseases. Brain Pathol 2006; 16: 143-154.

25. Wright LS, Prowse KR, Wallace K, Linskens MH, Svendsen CN. Human progenitor cells isolated from the developing cortex undergo decreased neurogenesis and eventual senescence following expansion in vitro. Exp Cell Res 2006; 312: 2107-2120.

26. Pluchino S, Zanotti L, Rossi B, Brambilla E, Ottoboni L, Salani G et al. Neurosphere-derived multipotent precursors promote neuroprotection by an immunomodulatory mechanism. Nature 2005; 436: 266-271.

27. Song $\mathrm{H}$, Stevens $\mathrm{CF}$, Gage $\mathrm{FH}$. Astroglia induce neurogenesis from adult neural stem cells. Nature 2002; 417: 39-44.

28. Kaushal D, Contos JJ, Treuner K, Yang AH, Kingsbury MA, Rehen SK et al. Alteration of gene expression by chromosome loss in the postnatal mouse brain. J Neurosci 2003; 23: 5599-5606.

29. Maslov AY, Barone TA, Plunkett RJ, Pruitt SC. Neural stem cell detection, characterization, and age-related changes in the subventricular zone of mice. J Neurosci 2004; 24 1726-1733.

30. Rehen SK, Yung YC, McCreight MP, Kaushal D, Yang AH, Almeida BS et al. Constitutiona aneuploidy in the normal human brain. J Neurosci 2005; 25: 2176-2180.
31. Yang AH, Kaushal D, Rehen SK, Kriedt K, Kingsbury MA, McConnell MJ et al. Chromosome segregation defects contribute to aneuploidy in normal neural progenitor cells. J Neurosci 2003; 23: 10454-10462.

32. Yurov YB, lourov IY, Vorsanova SG, Liehr T, Kolotii AD, Kutsev SI et al. Aneuploidy and confined chromosomal mosaicism in the developing human brain. PLOS ONE 2007; 2 e558.

33. Bailey KJ, Maslov AY, Pruitt SC. Accumulation of mutations and somatic selection in aging neural stem/progenitor cells. Aging Cell 2004; 3: 391-397.

34. Carpenter MK, Cui X, Hu ZY, Jackson J, Sherman S, Seiger A et al. In vitro expansion of a multipotent population of human neural progenitor cells. Exp Neurol 1999; 158: 265-278.

35. Jain M, Armstrong RJ, Tyers $P$, Barker RA, Rosser AE. GABAergic immunoreactivity is predominant in neurons derived from expanded human neural precursor cells in vitro. Exp Neurol 2003; 182: 113-123.

36. Sah DW, Ray J, Gage FH. Regulation of voltage- and ligand-gated currents in rat hippocampal progenitor cells in vitro. J Neurobiol 1997; 32: 95-110.

37. Augustine GJ, Charlton MP, Smith SJ. Calcium action in synaptic transmitter release. Annu Rev Neurosci 1987; 10: 633-693.

38. Bading H, Ginty DD, Greenberg ME. Regulation of gene expression in hippocampal neurons by distinct calcium signaling pathways. Science 1993; 260: 181-186.

39. Campbell DT. Single-channel current/voltage relationships of two kinds of $\mathrm{Na}+$ channel in vertebrate sensory neurons. Pflugers Arch 1993; 423: 492-496.

40. Pozzan T, Rizzuto R, Volpe P, Meldolesi J. Molecular and cellular physiology of intracellular calcium stores. Physiol Rev 1994; 74: 595-636.

Supplementary Information accompanies the paper on Cell Death and Differentiation website (http://www.nature.com/cdd) 